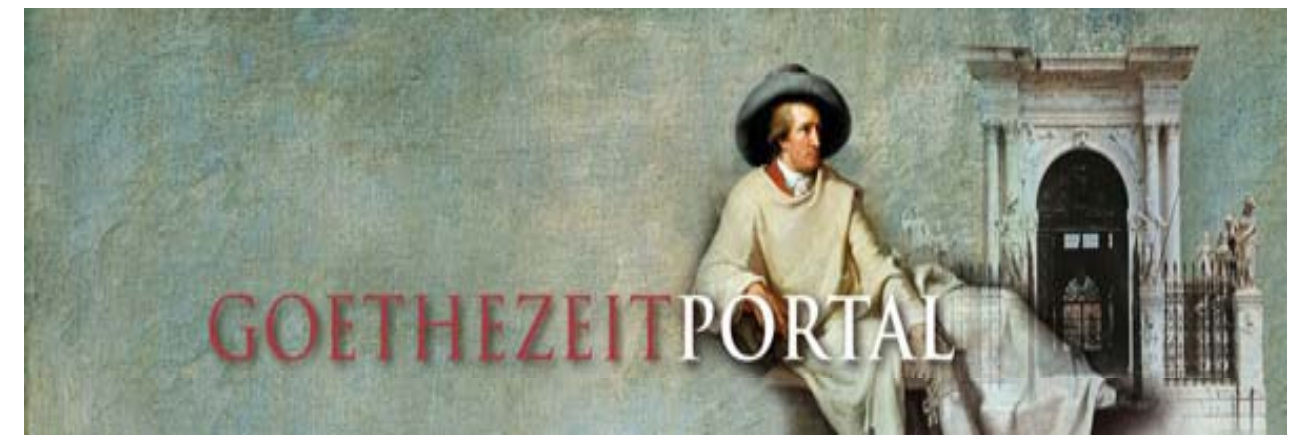

PETER HÜHN

\title{
Friedrich Hölderlin:
}

„Andenken“

\section{Erstpublikation:}

Jörg Schönert / Peter Hühn / Malte Stein: Lyrik und Narratologie. Text-Analysen zu deutschsprachigen Gedichten vom 16. bis zum 20. Jahrhundert (Narratologia. Contributions to Narrative Theory; 11) Berlin: de Gruyter 2007, S. 99-112.

Vorlage:

PDF-Datei des Autors.

Autor:

Prof. Dr. Peter Hühn

Universität Hamburg

Institut für Anglistik und Amerikanistik

Von-Melle-Park 6

20146 Hamburg

Homepage:

$<$ http://www.sign-lang.uni-hamburg.de/fb07/EnglS/Lehrende_H/H_huehn.htm> E-Mail:<Huehn@uni-hamburg.de> 


\section{Peter HüHN}

\section{Friedrich Hölderlin: „Andenken“}

(I)

Der Nordost wehet,

Der liebste unter den Winden

Mir, weil er feurigen Geist

Und gute Fahrt verheißet den Schiffern.

5 Geh aber nun und grüße

Die schöne Garonne,

Und die Gärten von Bordeaux

Dort, wo am scharfen Ufer

Hingehet der Steg und in den Strom

10 Tief fällt der Bach, darüber aber

Hinschauet ein edel Paar

Von Eichen und Silberpappeln;

(II)

Noch denket das mir wohl und wie

Die breiten Gipfel neiget

15 Der Ulmwald, über die Mühl',

Im Hofe aber wächset ein Feigenbaum.

An Feiertagen gehn

Die braunen Frauen daselbst

Auf seidnen Boden,

20 Zur Märzenzeit,

Wenn gleich ist Nacht und Tag,

Und über langsamen Stegen,

Von goldenen Träumen schwer,

Einwiegende Lüfte ziehen.

(III)

25 Es reiche aber,

Des dunkeln Lichtes voll,

Mir einer den duftenden Becher,

Damit ich ruhen möge; denn süß

Wär' unter Schatten der Schlummer.

30 Nicht ist es gut,

Seellos von sterblichen

Gedanken zu seyn. Doch gut

Ist ein Gespräch und zu sagen

Des Herzens Meinung, zu hören viel

35 Von Tagen der Lieb',

Und Thaten, welche geschehen.

(IV)

Wo aber sind die Freunde? Bellarmin

Mit dem Gefährten? Mancher

Trägt Scheue, an die Quelle zu gehn; 

Im Meere. Sie,

Wie Mahler, bringen zusammen

Das Schöne der Erd' und verschmähn

Den geflügelten Krieg nicht, und

$45 \mathrm{Zu}$ wohnen einsam, jahrlang, unter

Dem entlaubten Mast, wo nicht die Nacht durchglänzen

Die Feiertage der Stadt,

(V)

Und Saitenspiel und eingeborener Tanz nicht.

Nun aber sind zu Indiern

Die Männer gegangen

Dort an der luftigen Spiz'

An Traubenbergen, wo herab

Die Dordogne kommt,

Und zusammen mit der prächt'gen

55 Garonne meerbreit

Ausgehet der Strom. Es nehmet aber

Und giebt Gedächtniß die See,

Und die Lieb' auch heftet fleißig die Augen,

Was bleibet aber, stiften die Dichter.

Friedrich Hölderlin: „Andenken“, in: Friedrich Hölderlin: Sämtliche Werke. Bd. 2, hg. von Friedrich Beißner. Stuttgart 1951, S. 188f.

„Andenken“, Hölderlins vermutlich letztes vollendetes Gedicht, ${ }^{1}$ ist ein außerordentlich komplexer, viel interpretierter und kontrovers gedeuteter Text, der bisher mit Bezug auf unterschiedliche Problemkomplexe und Fragestellungen erörtert worden ist - beispielsweise unter (kunst-)philosophischen oder metaphysischen Perspektiven verschiedener Art (Heidegger [1981], Henrich [1986], Homann [1986], Gethmann-Siefert [1987], Henrich [1990]), unter geographisch-biographischen Aspekten (Henrich [1986], Lefebvre [1989]), im zeitgenössischen politischen Kontext (Gaier [1989]), als Rekonstitution des Mythos (Böschenstein [1984]) oder im poetologischen Rahmen (Hamlin [1987], Valk [2003], Martel [2004]). Diese thematischen Aspekte sind vielfach von sehr großer Abstraktheit und Allgemeinheit, und entsprechend herrscht eine Neigung vor, das Gedicht als poetologischen oder philosophischen Entwurf zu lesen. Die folgenden Ausführungen konzentrieren sich demgegenüber (aber mit Anschließbarkeit an einige bisherige Interpretationsansätze, wie etwa den von Martel [2004]) gemäß dem narratologischen Analysekonzept auf das

1 Entstanden 1803, Erstdruck 1808. 
Gedicht als Reflexionsprozess (und der schließlich damit verbundenen Reflexion von Dichtung) im Rahmen der Imaginations- und Erinnerungsthematik, die bereits mit der Überschrift „Andenken“ angesprochen wird. Auf eine ausführliche Auseinandersetzung mit anderen (insbesondere primär philosophisch-metaphysischen) Deutungsansätzen wird verzichtet.

\section{Zwei gegenläufige Sequenzen als Grundstruktur}

Der Gesamtaufbau des Gedichtes ist durch das Prinzip von einander gegenläufigen Strukturen bestimmt. In formaler Hinsicht ist der Text in fünf (reimlose, rhythmisch variable) Strophen gegliedert, von denen die ersten vier jeweils 12 Verse umfassen, abweichend dazu die fünfte Strophe jedoch nur 11 Verse. Aber in der Thematik und der Perspektivierung der Rede ist eine (nahezu symmetrische) Zweiteilung zu beobachten. In den ersten 29 Versen sind die Aussagen persönlich formuliert, die beschriebenen Vorgänge pronominal auf den Sprecher bezogen („,mir“, „ich“) und im Präsens-Indikativ oder im Imperativ, ohne Fragen oder Verneinungen abgefasst, das heißt die Vorgänge werden als sich unter Beteiligung des Sprechers vollziehend oder zu vollziehen gedacht. Die folgenden 29 Verse (V.30-58) präsentieren dagegen allgemein formulierte gnomische Sätze in Verbindung mit Aussagen über andere Personen ohne jeden pronominalen Bezug auf den Sprecher, mit Fragen, Verneinungen und auch im Präteritum, das heißt die beschriebenen Sachverhalte werden aus kognitiver Distanz und ohne Verbindung zum Sprecher thematisiert und reflektiert. Während also die erste Hälfte des Gedichtes die Welt stets auf das Ich bezieht, erscheint diese in der zweiten losgelöst und unabhängig von ihm.

Der Vers 59 in der letzten elf- statt zwölfzeiligen Strophe (in der also eigentlich ein Vers ,fehlt ${ }^{*}$ ) ist paradoxerweise bei der Zweiteilung (in zweimal 29 Zeilen) numerisch überzählig (als die 30. Zeile), und diese Zeile ist auch als explizite Aussage über Dichtung thematisch im gesamten Gedicht, nicht nur in seiner zweiten Hälfte (V.30ff.), isoliert und wird auf diese Weise besonders herausgehoben (s.u.).

Die beiden Teile des Gedichtes (V.1-29 u. 30-58) - unter Absehung von der Schlusszeile - lassen sich aufgrund der Aussageformen, die ihnen jeweils eine tendenziell einheitliche, aber einander kontrastierende Wahrnehmungsweise und Sprechhaltung verleihen, in zwei mentale Sequenzen gliedern. Sie lassen sich als zwei Abfolgen von je unterschiedlichen Bewusstseinszuständen, Wahrnehmungen von Sachverhalten und Artikulati- 
on von Lebenseinstellungen (als Geschehenselemente) rekonstruieren. ${ }^{2}$ Die stellenweise inkohärent wirkende Aufeinanderfolge dieser Bewusstseinszustände kann durch die Beschreibung von Isotopien in ihren semantischen Zusammenhängen und Bewegungsrichtungen deutlich gemacht werden. Es handelt sich, kurz zusammengefasst, um zwei gegenläufige imaginative Bewegungen in der Konstellation von Ich und Welt, die Bewegung nach Innen und die nach Außen, wie im Folgenden im Einzelnen nachgezeichnet werden soll.

\section{Die erste Sequenz: Rückzug auf das Ich}

Die erste Sequenz bietet eine Reihe von Bildern aus der Hafenstadt Bordeaux und ihrer Umgebung, die offenbar Erinnerungen repräsentieren. Mit ihrer Hilfe versetzt sich der Sprecher aus der Ferne - aus dem nordöstlich hiervon gelegenen Deutschland, wie die Windrichtung (vgl. V.1) sowie die Biographie des Autors nahelegen - an diesen Ort (V.8: „,dort“) imaginativ wieder zurück (V.13: „,noch denket das mir wohl“). Diese gedankliche (Rück-)Versetzung wird über die Wahrnehmung des Nordostwindes inszeniert (V.11f.: „Der Nordost wehet / [...] / Geh aber nun und grüße / [...]"); ${ }^{3}$ sie löst einen imaginativen (Wieder-)Vergegenwärtigungsprozess aus, wie die durchgängige Verwendung des Präsens betont.

Dementsprechend ist der (thematische) Frame dieser Sequenz zunächst als - sehnsuchtsvolle - persönliche Erinnerung zu bestimmen, allgemeiner dann als Lebenseinstellung in der Relation von Ich und Welt. Der persönliche Bezug auf den Sprecher wird durch die wiederholte Verwendung des Pronomens der 1. Person (V.3 u. 13 sowie V.27f.) nachdrücklich und durchgängig herausgestellt. Dieser erinnernde (Rück-)Versetzungs- und

2 Zur Doppelperspektive vgl. grundsätzlich Martel (2004). Sein Ansatz, die Analyse von vornherein in einen (wie er es nennt), poesiologischen' Rahmen zu stellen, scheint mir die Signifikanz der plötzlichen Schlusswendung zum Dichter-Thema abzuschwächen und der Prozessstruktur des Gedichtes nicht gerecht zu werden. Die von Valk (2003) postulierte Grundopposition von Liebe und Tat, die in der Dichtung aufgehoben werde und dort ihre Dauer erhalte, basiert auf einer sehr forcierten Zuschreibung von Erotik für den Bedeutungszusammenhang der ersten Strophen. Sie ignoriert zudem den unterschiedlichen Rededuktus und den Unterschied in der Position des Ich und seiner Involviertheit in den thematisierten Sachverhalten.

3 Wie Jamme (1988) vorschlägt, kann man in der Anrufung des Windes eine Anspielung auf das Konzept der Inspiration erblicken - eine Anspielung, so lässt sich hinzufügen, die den imaginativen Charakter der folgenden Bilder unterstreicht (S. 648). 
Imaginationsprozess unterliegt einem spezifischen Ablauf und einer Tendenz zur Veränderung, die sich isotopisch vor allem in den räumlichen und visuellen Komponenten der vermittelten Bilder, also in den Semen der dafür verwendeten Wörter manifestiert, nämlich als Bewegung von oben nach unten sowie als Verschiebung von Dynamik und Aktivität zu Langsamkeit, Ruhe und harmonischer Balance. ${ }^{4}$ Besonders klar tritt eine derartige Entwicklungstendenz in Erscheinung beim Umschlag von dem (mit dem Nordostwind assoziierten) „feurigen Geist“ und der „gute[n] Fahrt“ (V.3f.) am Anfang zu den von ,goldenen Träumen schwere[n]" ,über langsamen Stegen“ ziehenden „einwiegende[n] Lüfte[n]“ (V.22-24) sowie zu dem am Schluss dieser Sequenz ausgesprochenen Verlangen nach Herbeiführung von Ruhe und süßem „Schlummer“ mit Hilfe von Weingenuss (vgl. V.25-29).

Dieselbe Bewegung drückt sich subtiler aus im Fallen des Bachs nach unten in den Strom (V.9f.) verbunden mit dem ruhigen ,Darüber-Hinschauen' der Bäume, augenscheinlich ebenfalls nach unten (,darüber [...] hinschauet ein edel Paar / [...]“ - V.10-12), sowie im Neigen des Ulmenwaldes ,über die Mühl“ (V.14f.). In anderer Weise setzt sich diese Tendenz fort in den Implikationen von feiertäglicher Geruhsamkeit (V.17: „an Feiertagen“), von Gedämpftheit (V.19: „auf seidenen Boden“) und Ausgleich der Gegensätze im Frühlingsäquinoktium (,wenn gleich ist Nacht und Tag" - V.21), aber auch im Betonen wachsender Dunkelheit als visuellem Äquivalent von Ruhe und Abschirmung (V.27: „,des dunklen Lichtes voll“, V.29: „Schatten“).

Die sich hier vollziehende Bewegung stellt einen imaginierten Rückzug aus Weite, Geschäftigkeit und Aktivität zu Ruhe, Abgeschlossensein, Schlaf und Vergessen dar als Wandel in der psychischen Einstellung und als mentalen Veränderungsprozess beim Sprecher. Dass eine Veränderung eintritt, zeigt sich besonders prägnant in der Differenz bei gleichbleibender Subjektbezogenheit zwischen Anfang und Ende der Sequenz, nämlich zwischen einerseits den persönlichen Assoziationen von Wind und Dynamik (V.2-4: „Der liebste unten den Winden / Mir, weil er feurigen Geist / Und gute Fahrt verheißet [...]") und den Aufforderungen zum Aufbruch in die Weite (V.5: „Geh aber nun und grüße / [...]") und andererseits der abschließenden Äußerung des Wunsches nach Ruhe (V.25ff.: „Es reiche aber / [...] / Damit ich ruhen möge“), dessen Intensität durch die unmittelbare Koppelung von „ich“ als Subjekt und dem Verb „ruhen“, der einzigen Verwendung des Personalpronomens der

4 Vgl. Martel (2004), S. 387ff. 
1. Person im Nominativ, eine besondere Emphase erhält. In dieser Verschiebung vom Wahrnehmen der Umwelt hin zur Wunschvorstellung für den künftigen Zustand des Sprechers ist die rudimentäre Form eines Skripts zu sehen, das in der Literatur um 1800 vielfach ausgearbeitet wurde (,Weg nach innen'). Die erste Sequenz setzt somit ein mit dem imaginativen Gang in die Ferne, nach Außen, um dann das Dämpfen und Auslöschen dieser Bilder und den Rückzug, die Regression ins Ich zu vollziehen und zum Abschluss zu bringen.

Die Kohärenz dieser Sequenz wird durch interne - mit der Adversativpartikel ,aber" markierte - Steigerungsschritte gegliedert und in ihrer Bewegungsrichtung akzentuiert. Mit „aber“ wird jeweils (insgesamt viermal) eine vorher beschriebene Tendenz mit einem anderen, gesteigerten Bild kontrastiert und graduell übertroffen - in Richtung auf größere Ruhe und stärkere Abgeschlossenheit. Dieser Kontrast nimmt folgende Formen der Verschiebung an: in Vers 5 von der dynamischen Ausfahrt über das weite Meer zu dem imaginativen Gang zu Fluss und Stadt mit (begrenzendem) Ufer und (umfriedeten) Gärten, in Vers 10 vom heftigen Fall des Baches zum stillen Schauen der Bäume, in Vers 16 von der Neigebewegung einer Vielzahl von Bäumen zum unmerklich-ruhigen fruchttragenden Wachstum eines Einzelbaums ${ }^{5}$ und von der (implizierten) Arbeitsaktivität einer Mühle zum (abgeschirmten, geschützten) Bezirk eines Hofes und in Vers 25 von diesen impliziten Bildern der Ruhe und Dämpfung zu der expliziten Artikulation der Sehnsucht nach Schlaf und Auslöschung.

Die narrative Qualität dieser ersten Sequenz liegt somit nicht auf der Ebene des Geschehens, sondern in der Verknüpfung der subjektbezogenen Wahrnehmung der Außenwelt und der Wunschvorstellungen zum Zustand des Sprechers. Dadurch entsteht der Eindruck einer Bewusstseinsveränderung im Sprecher: die regressive Veränderung wird quasidramatisch inszeniert und in der Aneinanderreihung sich qualitativ wandelnder Erinnerungsbilder und Wunschvorstellungen vollzogen.

5 Die Bedeutung des Feigenbaums ist in diesem Sinne weiter spezifizierbar, wenn man hier eine Anspielung auf seine Symbolfunktion im Alten Testament für das erfüllte glückselige Leben im Reich Gottes sieht (1 Kön 4, 5; Mi 4, 4; Sach 3, 10; Joel 2, 21f.) - übrigens stets in Verbindung mit dem Weinstock, siehe Martel (2004), S. 388. Darüber hinaus scheint der Feigenbaum für Hölderlin mit Dionysos assoziiert zu sein - vgl. Gaier (1989), S. 180f. - und antizipiert somit den diese Sequenz abschließenden Verweis auf den Becher Wein (vgl. V.27). 


\section{Die zweite Sequenz: Aufbruch in die Welt}

Mit einer abrupten Abkehr von der Tendenz zum Rückzug und zur Konzentration auf das eigene Ich und zu dessen schließlicher Auslöschung als Ergebnis dieser ersten Sequenz wird dann innerhalb der dritten Strophe (also konträr zum formalen Aufbau) unvermittelt eine genau gleichlange zweite Sequenz (V.30-58) eingeführt, die eine gegenläufige Bewegung hin zu Progress, Aufbruch in die Weite, Öffnung nach Außen und Wachheit umfasst. Der Gegensatz der beiden Sequenzen wird durch verschiedene direkte Bezugnahmen und durch Umkehrung der wieder aufgegriffenen Motive zusätzlich betont. Endet die erste Sequenz im Verlangen nach Auslöschung des Bewusstseins im Schlaf, so setzt die zweite ein mit der ausdrücklichen Abwertung des Wunsches nach Rückzug aus ,der Welt' und der menschlichen Gemeinschaft auf das eigene Ich, ,seellos von sterblichen / Gedanken zu seyn" (V.31f.), ${ }^{6}$ und fährt mit der positiven Bestimmung der Alternative fort: „Doch gut / Ist ein Gespräch und zu sagen / Des Herzens Meinung, zu hören viel / Von Tagen der Lieb, / Und Thaten, welche geschehen“ (V.32-36). Die Richtung kehrt sich um: Ausdruck und Austausch (statt Regression und Vergessen), Wahrnehmung der Welt in der Vielheit ihres Geschehens.

Die Gegensätzlichkeit der beiden Sequenzen zeigt sich vornehmlich in dem Kontrast zwischen der Ichbezogenheit (dem Rückzug auf das Ich) der ersten und dem Absehen vom Ich in der zweiten, zwischen Regress und Progress, zwischen Individualität und Isolation auf der einen Seite und Gemeinschaftlichkeit und Kommunikation auf der anderen: ${ }^{7}$ Die Perspektive richtet sich jetzt auf die Welt außerhalb der Selbstwahrnehmung des Ich. Zugleich impliziert dieser Kontrast auch, dass der Sprecher mit der ersten Sequenz sich selbst identifiziert, die zweite jedoch prononciert mit anderen Personen verbindet - als Beispiele werden „die Freunde“"

6 Diese Wendung ist sehr unterschiedlich und tiefsinnig gedeutet worden; vgl. z.B. den Überblick bei Jamme (1988), S. 650; siehe auch Reuß (1990), S. 240ff. Aber durch die direkt antithetische Position zu dem unmittelbar zuvor formulierten Ziel der ersten Sequenz, dem Verlangen nach Vergessen, wird primär die Bedeutung der Bewusst- und Fühllosigkeit, der Auslöschung von Erinnerungen und Gedanken an das Vergängliche (das „Sterbliche“) nahegelegt. Der Ausdruck „seellos“ charakterisiert ein solches Verlangen sogleich als negativ. In dieser Abwertung schwingt die schwäbische Bedeutung des Wortes (im Sinne von ,ruchlos“) mit, auf die Binder (1986), hinweist (S. 16).

7 Vgl. grundsätzlich Martel (2004), der allerdings den Analogien beider Einstellungen gegenüber den Differenzen zu viel Gewicht gibt (S. 386ff.). 
„Bellarmin mit dem Gefährten“ (V.37f.) ${ }^{9}$ genannt, auf die anschließend offenbar mit „sie“ (V.41) Bezug genommen wird und die später mit den „Männer[n]“ (V.50) assoziiert werden. Während das Ich in der ersten Sequenz anwesend ist und das Geschehen ausschließlich auf sich bezieht, sind die Bezugspersonen der zweiten Sequenz bezeichnenderweise abwesend, das heißt vom Sprecher entfernt - sie sind in die Welt (vgl. V.41ff.), zum Beispiel nach Indien (vgl. V.49ff.), gegangen, und zwar schon in der Vergangenheit, so dass die Distanz zum Sprecher (und zu seiner Haltung in der ersten Sequenz) sich sowohl auf der räumlichen als auch zeitlichen Ebene manifestiert.

Die Bewegung nach außen wird in der vierten und fünften Strophe durch Wiederaufnahme des Meeres-Motivs aus der ersten Sequenz weiter profiliert. Löst sich der Sprecher in der ersten Sequenz schließlich vom Nordostwind ab, der sich vom Festland zum Meer hin bewegt, und hält in Bordeaux und an der Garonne inne, so geht die Bewegung der zweiten Sequenz nun explizit und entschieden in die Weite über die (Land-) „Spitze“ (V.51) hinaus, bei der sich Garonne und Dordogne zur meerbreiten Gironde vereinigen und in den Ozean münden. Das prononciert wiederholte Ortsadverb „dort“ im Zusammenhang mit Bordeaux macht diesen Gegensatz der räumlichen Orientierung (Innehalten vs. Aufbruch) besonders sinnfällig (V.8 vs. V.51). Während die Bewegung der ersten Sequenz durch Konzentration, Reduktion, Kontraktion und Rückzug charakterisiert ist, verläuft die Linie in der zweiten nicht zurück an die Quelle (diese scheint vielmehr mit den Erfahrungen von Kontraktion und Konzentration verbunden zu sein), ${ }^{10}$ sondern entgegengesetzt auf das Meer hinaus, in das die Flüsse münden: Gerade das Meer ist mit Reichtum und Vielfalt (V.40f.) assoziiert (und nicht die Quelle), daher „die Scheue“

8 Mit den Freunden mögen die des Sprechers gemeint sein, aber der Bezug auf ihn wird gerade gekappt - durch das fehlende Possessivpronomen und durch ihre Entfernung.

9 Dies ist eine deutliche Anspielung auf Hölderlins Roman Hyperion oder der Eremit in Griechenland (1797/99). Mit diesem Bezug sowie mit der Nennung Indiens als dem Reiseziel der Männer erhält der Gang nach Außen und in die Welt eine implizit politische Dimension, im Sinne des Freiheitskampfes (zu den politischen Anspielungen im Zusammenhang mit Indien siehe unten S. 107); vgl. dazu Gaier (1989), S. 188ff.

10 Auch dieser Begriff ist sehr unterschiedlich interpretiert worden; z.B. sieht Reuß (1990) keinen Gegensatz zwischen Quelle und Meer, sondern eine paradoxale Einheit (S. 264ff.). Die Syntax signalisiert jedoch einen Kontrast zweier Verhaltensweisen (,an die Quelle zu gehen' vs. ,auf das Meer hinauszufahren') und nennt die Begründung für die Bevorzugung der zweiten Alternative (Reichtum, sinnliche Fülle ist allererst im Meer zu finden); vgl. hierzu Henrich (1986), S. 100ff.; auch Gaier (1989), S. 180f. 
(V.39), die mangelnde Bereitschaft, an die Quelle ${ }^{11}$ (zurück) zu gehen. Dieser Reichtum, der durch ,Aktion' zu erlangen ist, wird im Folgenden (V.41ff.) beispielhaft konkretisiert - als Schönheit der Erde und als „geflügelter" (also dynamischer, belebender) Krieg (V.44). Das aktive Leben ist aber auch mit Entbehrungen und Gefahren verbunden. Diese Ambivalenz wird beispielhaft im Verweis auf die „Indier“ (V.49) angesprochen: Zum einen galt Indien (damit wäre gleichermaßen Ost- wie Westindien gemeint) allgemein als legendäres Land reicher Schätze, zum anderen lässt sich in Indien (als Westindien) eine spezielle Anspielung auf Amerika und seinen Freiheitskampf sehen (zu dessen Unterstützung der Marquis de Lafayette 1776 von Bordeaux aus nach Südwesten, also in der Richtung des Nordostwindes, aufgebrochen war). ${ }^{12}$ Die mit der Öffnung in die Welt verbundene Aktivität wird dann - wiederum im kontrastierenden Rückgriff auf ein Motiv der ersten Sequenz, nämlich die „Feiertage der Stadt" (V.47 vs. V.17) - eigens hervorgehoben: Für diese Lebensorientierung gibt es keine Feiertage als Zeit der festlichen Muße und des Ausruhens vom Handeln. Einen Kontrast zur Reife und ruhigen Erfüllung markiert auch der „entlaubte Mast“ (V.46) - im Gegensatz zu den blättertragenden Bäumen der ersten Sequenz. Die Seefahrer werden zudem mit Malern verglichen (vgl. V.42), wobei als ,tertium comparationis ${ }^{\circ}$ das Sammeln, Einbringen und Festhalten der konkreten Schönheiten der Welt benannt und die Sinnlichkeit solcher Erfahrungen unterstrichen wird (vgl. V.42ff.). Gerade mit Bezug auf die Motivik der Seefahrt trägt diese Lebenseinstellung Züge des Heroischen, im Gegensatz zur Idyllik, die den in der ersten Sequenz beschriebenen Schauplatz charakterisiert.

Die die Sequenz abschließende erste gnomische Aussage (V.56-58) vor dem letzten Vers! - stellt eine Art generalisierender Zusammenfassung über das der zweiten Sequenz zugrundeliegende Prinzip dar. Zum Einen wird auf die See, die Welt außerhalb des Ich, in ihrer umfassenden Ambivalenz des Gebens und Nehmens, also in ihrer riskanten, nicht kontrollierbaren Eigendynamik, verwiesen, und zwar mit Bezug auf das ,kollektive' Gedächtnis, das gedankliche Festhalten, das nicht in der

11 Der Gang zur Quelle und die Fahrt aufs Meer sind offenbar als gegensätzliche Lebenseinstellungen etwa im Sinne von, vita contemplativa' und, vita actica' zu verstehen. Aber die Quelle dient hier vor allem als Kontrastbegriff zum Meer und nicht der Charakterisierung der Lebensform des Sprechers - denn dessen Regress und Wunsch nach Auslöschung ist keineswegs die Entscheidung für die Kontemplation.

12 Vgl. Gaier (1989), S. 178f. u. 189ff. In dem Verweis auf die Indier werden Assoziationen von Kolumbus und Lafayette, von Reichtum und Freiheit miteinander verknüpft. 
Macht des Einzelnen liegt - im Gegensatz zur ersten Sequenz, wo der Sprecher durch Imperative und konstative Aussagen die Erinnerung zu steuern vermochte. Einen eklatanten Kontrast zur ersten Sequenz stellt ebenfalls die folgende Wendung her: „die Lieb auch heftet fleißig die Augen" (V.58). Im Gegensatz zum ersehnten Schlummer (vgl. V.29), bei dem die Augen naturgemäß geschlossen sind, wird jetzt die intensive (,fleißig[e]“) Wachheit und Aufmerksamkeit der Sinne auf das Andere, auf die außerhalb des Bewußtseins existente Welt, hervorgehoben. Die „Lieb'“ öffnet das Ich für das Andere, setzt ,Ich“ und ,Welt' miteinander in Beziehung. ${ }^{13}$

In zwei Passagen auch dieser zweiten Sequenz markiert die Adversativpartikel „aber“ wiederum den Steigerungsprozess innerhalb des Ablaufs an besonders signifikanten Stellen und verdeutlicht so die Kohärenz. In Vers 49 wird die Frage nach dem Verbleib der Freunde (V.37ff.) beantwortet: Wie der Strom sich in die Wasserfülle des Meeres ergießt, so sind die Freunde hinaus, in die Welt ${ }^{6}$ aufgebrochen. In Vers 56 wird aus den vorausgegangenen konkreten Beispielen der Bewegung nach Außen das abstrakte Fazit gezogen: Für das Erinnern, das Bewahren von ,Andenken' an die aktive Existenz in der Welt, lässt sich keine sichere Prognose stellen.

Allgemeiner Frame der zweiten Sequenz ist - ähnlich wie bei der ersten - das Thema der Lebenseinstellung hinsichtlich des Verhältnisses von Ich und Welt, und als abstraktes Verlaufsmuster, als rudimentäres Skript läßt sich hier der Prozess der Öffnung gegenüber der Welt, des Sammelns von Erfahrungen jenseits des eigenen Ich benennen, jedoch verbunden mit dem Verlangen nach Festhalten und Bewahren. In dieser Lebenseinstellung begibt sich das Ich in die Welt, um sie sich anzueignen und so seine Existenz zu erweitern. Das dafür notwendige bewahrende „Gedächtniß“, das Dauer verleihende ,Andenken“, ist jedoch ein prekärer Vorgang, weil es nicht der Kontrolle des Einzelnen unterliegt, sondern dem Willen und der Macht der „See“, der sich entziehenden oder darbietenden Welt, dem Leben.

Der narrative Status der zweiten Sequenz ist anders angelegt als der der ersten. Vollzieht sich dort die narrative Veränderung auf der Darbietungsebene, also in der Bewusstseinsdimension, als ein Einstellungswandel des Sprechers, so hier auf der Geschehensebene als ein Handeln anderer Figuren, der Freunde des Sprechers. Während ferner die erste Se-

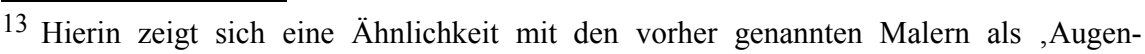
menschen'. 
quenz den narrativen Prozess weitgehend kontinuierlich in den imaginierten Bildern präsentiert, koppelt die zweite nicht-narrative, gnomische Passagen (V.30-32, 38-41 u. 56-58) mit kurzen, komprimierenden narrativen Abschnitten (dem gesprächsweisen Austausch von Meinungen und Erfahrungen in den Versen 33 bis 36, der Aktivität der Seefahrer und Maler im Sammeln von Erfahrungen und Bildern in der Welt in den Versen 41 bis 48, dem Aufbruch der „Männer“ zur Reise von Bordeaux nach Indien in den Versen 49 bis 56).

\section{Die Schlusszeile: ereignishafter Umbruch in die Dichtungsthematik}

Das Verhältnis von Ich und Welt ist somit als genereller Frame beiden Sequenzen gemeinsam, aber für das Gestalten dieser Relation werden polar gegensätzliche Skripts gewählt: im (narrativen) Ausarbeiten des IchBezugs bzw. des Welt-Bezugs, in der Sehnsucht nach Abschließung gegenüber dem Verlangen nach Öffnung, in der Konzentration auf sich selbst gegenüber der Kommunikation und Kooperation in der Gemeinschaft, im Streben nach Ruhe und Auslöschen des Bewusstseins gegenüber dem Streben nach Aktivität und Erfahrungserweiterung. Beide Sequenzen sind zusätzlich in einen übergreifenden Frame eingefügt: Sie verbindet das Thema der Erinnerung - genauer des Bewahrens im Gedächtnis. Diese Konstellation wird zum Einen mit dem Titel „Andenken“ aufgerufen, zum Andern ist sie gleichermaßen in den beiden Sequenzen thematisiert. Während die erste Sequenz die Erinnerung (als bewusst geschaffene Imagination im performativen Sprechakt) inszeniert, das heißt in ihrer Entstehung und in ihrem Ablauf konkret vorführt, spricht die zweite Sequenz von Erinnerung begrifflich abstrakt und zusammenfassend: „zu hören viel / Von Tagen der Lieb, / Und Thaten, welche geschehen“ (V.34-36), „Sie [die Freunde / Schiffer] / Wie Mahler, bringen zusammen / Das Schöne der Erd' [...]“ (V.41ff.) und das „Gedächtniß“, das die See nimmt und gibt (V.56f.). Gemeinsam ist beiden Sequenzen in dieser Hinsicht, dass die Erinnerung nicht dauerhaft bewahrt werden kann. Während die erste Sequenz am Ende mit der Bewusstseinsaufgabe (im Schlaf) auch das Erinnerte auslöscht (vgl. V.28f.), endet die zweite Sequenz mit der Aussage, dass die See das Gedächtnis sowohl gebe als auch nehme (vgl. V.56-58). ,Nehmen“ erscheint hier zwar weniger definitiv als der Regress, der in der ersten Sequenz gewünscht wird (zumal das 
Geben und nicht das Nehmen die zweigliedrige Wendung abschließt), aber dennoch ist das Unsichere der erinnernden Bewahrung betont.

Hiergegen wird nun unvermutet in der Schlusszeile des Gedichts das Vertrauen in das bleibende Bewahren von Erfahrungen des Lebens durch die Dichter gestellt: „Was bleibet aber, stiften die Dichter“ (V.59). Diese Behauptung ist wie die gnomische Rede in Vers 56 durch die Adversativpartikel ,aber“ abgesetzt, jedoch nicht im Sinne der Steigerung wie zuvor, sondern des klaren Gegensatzes. Sie bildet den Kontrapunkt zu den Tendenzen in beiden Sequenzen, indem sie die bewusstseinsmäßige Entwicklungslinie der ersten Sequenz (das Auflösen von Erinnerung) und die sich an Aktionen anschließende Ungewissheit für das Erinnern der „Thaten“ (V.36) auf der übergeordneten Ebene negiert - im Verweis auf die Erinnerungskompetenz „der Dichter“. Indem hierin ein Bruch der in beiden Sequenzen am Schluss aufgerufenen Erwartung liegt, handelt es sich um ein Ereignis.

Schärfer formuliert: Da beide Sequenzen abgeschlossen schienen und so keine Fortsetzung zu erwarten war, wirkt die Replik der antithetischen Schlusszeile umso überraschender. Das „Stiften“ von Dauer durch Dichtung (oder genauer: die Behauptung eines derartigen Vermögens) ist ereignishaft als überraschende ,Erweiterung' (,Fortsetzung ${ }^{6}$ ) der beiden gegenläufigen, aber in den Aussagen zum möglichen Verlust von ,Andenken' übereinstimmenden Sequenzen. Der Grad der Ereignishaftigkeit wird noch durch den Umstand weiter verstärkt, dass ,Dichter" vorher nicht erwähnt worden waren und mit ihnen also ein neues Element - auch in Absetzung von den im Vergleich genannten anderen Künstlern, den Malern (vgl. V.42) - unvorbereitet eingeführt wird. ${ }^{14}$

Zuordnung und Status dieses Ereignisses sind komplex und mehrschichtig. Auf der einen Seite schließt die Behauptung gewissermaßen insofern an die zweite Sequenz an, als sie den Dichtern das Ermöglichen von Dauer in der Welt - im Gegensatz zur wechselvollen Existenz der Seefahrer (und der See) - zuschreibt. In diesem Sinne liegt ein Geschehensereignis vor. Auf der anderen Seite setzt die Schlusszeile semantisch

14 Jamme (1988) konstruiert einen wesentlichen Zusammenhang aller drei Schlusssätze (V.56-59) als eine dreifache Entfaltung des Andenkens und übersieht damit die internen Differenzen, die das Gedicht durch den oben beschriebenen Perspektivwechsel in der Konstellation Ich-Welt in den beiden Sequenzen strukturieren (S. 652). Auch Henrich (1986) betont den grundsätzlichen Zusammenhang der Schlusssätze als Formulierung der Ganzheit des bewussten Lebens und Entfaltung des modernen Bewusstseins (z.B. S. 118f., 135 u. 182). 
nicht den Darstellungsgang der zweiten Sequenz fort, sondern fügt unvermittelt ein neues, andersartiges Element an, was einen Anschluss damit nicht auf der Geschehens-, sondern auf der Darbietungsebene konstituiert. So betrachtet handelt es sich um ein Darbietungsereignis, denn die Dauerhaftigkeit wird nicht erzählt, sondern durch die gnomische Formulierung in der Komposition des Gedichtes postuliert und durch die Existenz des Textes als Gedicht realisiert. Ein Darbietungsereignis konstituiert die Schlusszeile demgemäß in der Hinsicht, dass sich ihre Aussage auch auf das vorliegende Gedicht und die Aktivität des Autors beziehen läßt. Für das Gedicht „Andenken“ wird seine abschließende Behauptung durch seine bloße Existenz eingelöst: ${ }^{15}$ Sowohl die Vergänglichkeit der Erinnerungsprozesse der ersten als auch die prekären Erfahrungsprozesse der zweiten Sequenz werden durch diesen Text dauerhaft festgehalten. Ein derartiger Rückbezug der innertextlichen Aussage auf die Textgestalt im Sinne eines Darbietungsereignisses ist - in unterschiedlichen Ausprägungsformen - typisch für poetologische Gedichte, für Gedichte, die den Dichtungsvorgang thematisieren. ${ }^{16}$

\section{Literatur}

Binder, Wolfgang

1986 Hölderlin: „Andenken“, in: Uvo Hölscher (Hg.): Turm-Vorträge 1985/86. Tübingen, S. 5-30.

Böschenstein, Bernhard

1984 Geschehen und Gedächtnis: Hölderlins Hymnen „Wie wenn am Feiertage ...“ und „Andenken“: Ein einführender Vortrag, in: Le pauvre Holterling: Blätter zur Frankfurter Ausgabe. Frankfurt a.M., S. 7-16.

Gaier, Ulrich

1989 Hölderlins vaterländischer Gesang „Andenken“, in: Hölderlin-Jb. 26, S. 175201.

Gethmann-Siefert, Annemarie

1987 Die „Poesie als Lehrerin der Menschheit“ und das „neue Epos“ der modernen Welt: Kontextanalysen zur poetologischen Konzeption in Hölderlins „Andenken“, in: Helmut Bachmaier / Thomas Rentzsch (Hg.): Poetische Autonomie?

15 Einen derartigen Rückbezug der Aussage des Gedichtes auf dieses selbst nimmt auch Martel (2004) vor, aber nicht als Selbst-Erfüllung, sondern als Aufforderung zum „poesiologischen“" Gespräch mit dem Leser (S. 404f.).

16 Beispiele aus der englischen Literatur finden sich etwa bei Coleridge, Keats und Wordsworth oder bei Yeats. Zu Beispielen aus der deutschen Lyrik siehe Hildebrand (2003) und Pott (2004). 
Zur Wechselwirkung von Dichtung und Philosophie in der Epoche Goethes und Hölderlins. Stuttgart, S. 70-100.

Heidegger, Martin

1981 Hölderlins Hymne „Andenken“. [1943], in: ders.: Gesamtausgabe. 1. Abt., Bd. 4, hg. von Curd Ochwadt. Frankfurt a.M., S. 79-151.

Henrich, Dieter

1986 Der Gang des Andenkens: Beobachtungen und Gedanken zu Hölderlins Gedicht. Stuttgart.

1990 Andenken, Erinnerung, Gedächtnis: Über Hölderlins Gedicht „Andenken“, in: Sinn und Form 42, S. 379-84.

Hildebrand, Olaf (Hg.)

2003 Poetologische Lyrik von Klopstock bis Grünbein. Gedichte und Interpretationen. Köln u.a.

Homann, Renate

1988 Das Besondere und das Allgemeine in der Dichtung. Anmerkungen zu Dieter Henrichs Buch: Der Gang des Andenkens. Beobachtungen und Gedanken zu Hölderlins Gedicht. Stuttgart 1986, in: Zeitschrift für philosophische Forschung 42, S. 620-644.

Hölderlin, Friedrich

1951 Sämtliche Werke. Große Stuttgarter Ausgabe, hg. von Friedrich Beißner. Bd. 2. Stuttgart.

Jamme, Christoph

1988 Hölderlin und das Problem der Metaphysik: Zur Diskussion um „Andenken“, in: Zeitschrift für philosophische Forschung 42, S. 645-665.

Lefebvre, Jean-Pierre

1989 Auch Stege sind Holzwege, in: Hölderlin-Jb. 26, S. 202-223.

Martel, Christoph

2004 Noch denket das mir wohl ... Poetik der Erinnerung in Hölderlins Hymnen „Andenken“ und „Mnemosyne“, in: Euphorion 98, S. 385-406.

Pott, Sandra

2004 Poetiken. Poetologische Lyrik, Poetik und Ästhetik von Novalis bis Rilke. Berlin / New York.

Reuß, Roland

1990 „... die eigene Rede des andern“: Hölderlins „Andenken“ und „Mnemosyne“. Basel / Frankfurt a.M.

Valk, Thorsten

2003 Das dunkle Licht der Dichtung. Zur Kunst des Erinnerns in Friedrich Hölderlins Hymne „Andenken“, in: Olaf Hildebrand (Hg.): Poetologische Lyrik von Klopstock bis Grünbein. Gedichte und Interpretationen. Köln u.a., S. 100-113. 